\title{
Microtensile bond strength of CAD/CAM materials to dentin under different adhesive strategies
}

\section{Mariá Cortina BELLAN(a) \\ Patrícia Fernandes Jerzewski \\ Sotero da CUNHA ${ }^{(b)}$ \\ Julieta Gomes TAVARES(a) \\ Ana Maria SPOHR ${ }^{(a)}$ \\ Eduardo Gonçalves MOTA(a)}

(a)Pontifícia Universidade Católica da Universidade Federal do Rio Grande do Sul - PUC-RS, School of Dentistry, Graduate Program, Porto Alegre, RS, Brazil.

(b)São Leopoldo Mandic, School of Dentistry, Graduate Program, Porto Alegre, RS, Brazil.

Declaration of Interests: The authors certify that they have no commercial or associative interest that represents a conflict of interest in connection with the manuscript.

\section{Corresponding Author:}

Eduardo Goncalves Mota

E-mail: eduardo.mota@pucrs.br

Submitted: July 21, 2017

Accepted for publication: Nov 8, 2017

Last revision: Nov 23, 2017
Abstract: The present study aimed to evaluate the microtensile bond strength ( $\mu \mathrm{TBS}$ ) of novel computer-aided design/computer-aided manufacturing (CAD/CAM) restorative materials to dentin using different adhesive strategies. Thirty-two crowns were milled using CAD/CAM materials (Vita Mark II as control, Vita Suprinity, Vita Enamic and Lava Ultimate) and luted to dentin using different resin cements (RelyX ARC, RelyX Unicem 2 and RelyX Ultimate). The specimens were stored in $100 \%$ relative humidity at $37^{\circ} \mathrm{C}$ for $24 \mathrm{~h}$ and sectioned. The samples $(n=16)$ with cross-sectional areas of approximately $0.90 \mathrm{~mm}^{2}$, were submitted to a $\mu \mathrm{TBS}$ test in a universal testing machine with a crosshead speed of $0.5 \mathrm{~mm} / \mathrm{min}$. The samples were analyzed with SEM to determinate the failure mode. According to 2-way ANOVA and Tukey's test $(\alpha=0.05)$, the interaction effect (material $x$ luting strategy) was significant $(\mathrm{p}=0.001)$. Regardless of the luting strategy, a higher $\mu$ TBS was obtained with Lava Ultimate and Vita Enamic, which were significantly different from Vita Mark II and Vita Suprinity $(p<0.05)$. For Vita Mark II, Vita Suprinity, Vita Enamic, and Lava Ultimate, the $\mu$ TBS obtained with RelyX Unicem 2 was not significantly different from that obtained with RelyX ARC or RelyX Ultimate. All groups obtained at least three types of failure. The adhesive strategy with self-adhesive resin cement was comparable to conventional resin cement with total-etch or self-etch adhesive techniques in the bond of novel CAD/CAM materials to dentin.

Keywords: Dental Bonding; Tensile Strength; Dentin; Resin Cements.

\section{Introduction}

The use of computer-aided design/computer-aided manufacturing (CAD/CAM) technology has become popular during recent decades in dentistry. This technology allows the dentist to mill restorations in a single visit, merging features, such as speed and easy handling, with longevity. ${ }^{1}$ The restorations can be milled using ceramics (feldspar, leucite, lithium-based, zirconia and alumina), ${ }^{2}$ a polymer-infiltrated ceramic network and a composite resin. ${ }^{3,4}$

To associate ceramic characteristics (high aesthetics, wear resistance, biocompatibility, and color stability) with those of composites (viscoelastic behavior and less wear of the opposite arch) and to improve its properties, 
new materials with different compositions have been recently introduced in the market for use in CAD/CAM systems. . $^{3,5}$,

Within these materials, Lava Ultimate (3M ESPE, St. Paul, MN, USA) is a nanoceramic resin that is composed of nanoceramic particles embedded in a highly cross-linked resin matrix, ${ }^{3,4}$ and Vita Enamic (VITA Zahnfabrik, Bäd Sackingen, Germany) is a polymer-infiltrated ceramic network that consists of a feldspar ceramic network infiltrated with a dimetacrylate polymer network. ${ }^{78} \mathrm{An}$ additional material that became available for CAD/CAM technology is Vita Suprinity (VITA Zahnfabrik, Bäd Sackingen, Germany). This material consists of a zirconiareinforced lithium silicate ceramic that supports a wide range of applications, and after crystallization, exhibits higher mechanical properties., ${ }^{9,1,11}$

Adhesive luting is recommended to bond CAD/CAM materials to the tooth structure and support the oral environment. However, the total-etch adhesive strategy is a complex, multi-step technique and may compromise the effectiveness of bonding. Due to this limitation, self-adhesive resin cements were developed to simplify the bonding procedures, to reduce clinical steps and to shorten the "window of contamination". These luting materials do not require any pretreatment of the tooth surface, such as an etchant, primer, or bonding agent; thus, the cementation can be performed in a single step. ${ }^{12,13}$

Due to the countless variables widely described in the literature, doubts about the interactions between the luting cement, restorative material, and conditioning protocol treatment remain in clinical practice. The present study aimed to evaluate the $\mu$ TBS of novel CAD/CAM restorative materials to dentin using three different adhesive strategies: i) conventional resin cement with the total-etch adhesive technique; ii) conventional resin cement with the self-etch adhesive technique; and iii) self-adhesive resin cement. The null hypothesis to be tested was that the adhesive strategies do not influence the bonding of $\mathrm{CAD} / \mathrm{CAM}$ materials to dentin.

\section{Materials and Methods}

\section{Specimen preparation}

Thirty-two intact caries-free extracted human third molars were selected for this study. All teeth were stored in an aqueous solution of $0.5 \%$ chloramine-T at $4^{\circ} \mathrm{C}$ for 7 days and then stored in distilled water at $4^{\circ} \mathrm{C}$ for a maximum of 6 months. The teeth were collected under a protocol reviewed and approved by the local ethics committee (Approval \# 48466815.7.0000.5336). Flat coronal dentin surfaces were exposed by removing the occlusal enamel and superficial dentin with a slow-speed, water-cooled diamond saw (Labcut 1010, Exterc Corp., London, England). Dentin surfaces were abraded with \#600 grit silicon carbide $(\mathrm{SiC})$ paper under running water to create standardized smear layers and then ultrasonically cleaned in distilled water for $5 \mathrm{~min}$.

The flat surfaces were coated with CEREC Optispray (Cerec Optispray, Sirona, Bensheim, Germany) and scanned with CEREC ${ }^{\circledR}$ Omnicam (Cerec Optispray, Sirona, Bensheim, Germany). A telescopic crown was designed based on the exact virtual 3D model reproduced. The crowns were milled according to the manufacturer's instructions using a Cerec MC XL (Cerec Optispray, Sirona, Bensheim, Germany).

The materials used in this study are presented in Table 1 . The specimens were divided into 12 groups according to the material (Vita Mark II as control, Vita Suprinity, Vita Enamic and Lava Ultimate) and luting cement (RelyX ARC, RelyX Unicem 2 and RelyX Ultimate).

\section{Surface treatment of the restorative materials}

Vita Mark II, Vita Suprinity and Vita Enamic: sandblasted with 50- $\mu \mathrm{m}$ aluminum-oxide $\left(\mathrm{Al}_{2} \mathrm{O}_{3}\right)$ particles with a dental airborne-particle abrasion unit (Bioart, São Carlos, Brazil); ultrasonically cleaned for 5 min in distilled water and air dried; etched with hydrofluoric acid 10\% (FGM, Joinville, Brazil) for $60 \mathrm{~s}$, $20 \mathrm{~s}$ and $60 \mathrm{~s}$, respectively; and then ultrasonically cleaned for $5 \mathrm{~min}$ in distilled water and air dried.

Lava Ultimate: sandblasted with $50-\mu$ m aluminumoxide $\left(\mathrm{Al}_{2} \mathrm{O}_{3}\right)$ particles and ultrasonically cleaned for $5 \mathrm{~min}$ in distilled water and air dried.

After, the silane coupling agent (Dentsply, Petrópolis, Brazil) was applied for $60 \mathrm{~s}$ and air dried for RelyX ARC and RelyX Unicem 2 luting cements. For RelyX Ultimate cement, a coating of Scotchbond Universal was applied and gently air-dried. 
Table 1. Materials, composition, batch number and, manufacturer.

\begin{tabular}{|c|c|c|}
\hline Material & Product and composition* & Batch \# \\
\hline Vita Mark II ${ }^{a}$ Control & $\begin{array}{c}\text { Silicon dioxide } 56-64 \% \text {, aluminum oxide } 20-23 \% \text {, sodium oxide } 9-11 \% \text {, potassium oxide } 6-8 \% \text {, calcium } \\
\text { oxide } 0.3-0.6 \% \text {, titanium dioxide } 0.0-0.1 \%\end{array}$ & 45500 \\
\hline$V_{\text {Vita Suprinity }}^{a}$ & $\begin{array}{c}\text { Zirconia-reinforced lithium silicate ceramic, zirconium oxide } 8-12 \% \text {, silicon dioxide } 56-64 \% \text {, lithium } \\
\text { oxide } 15-21 \% \text {, various }>10 \%\end{array}$ & 48940 \\
\hline Vita Enamic ${ }^{a}$ & $\begin{array}{l}\text { Hybrid ceramic (resin infiltrated ceramic network) Ceramic: silicon dioxide } 58-63 \% \text {, aluminum oxide } \\
20-23 \% \text {, sodium oxide 9-11\%, potassium oxide 4-6\%, boron trioxide } 0.5-2 \% \text {, zirconia and calcium } \\
\text { oxide. Polymer part (25\%): UDMA and TEGDMA }\end{array}$ & 45810 \\
\hline Lava Ultimate ${ }^{b}$ & $\begin{array}{l}\text { Cured dental restorative, consisting of silica nanomers }(20 \mathrm{~nm}) \text {, zirconia nanomers }(4-11 \mathrm{~nm}) \text {, } \\
\text { nanocluster particles derived from the nanomers }(0.6-10 \mathrm{~nm}) \text {, silane coupling agent, and resin matrix } \\
\text { (BisGMA, Bis-EMA, UDMA and TEGDMA). }\end{array}$ & N708503 \\
\hline \multirow{2}{*}{ RelyX ARC ${ }^{b}$} & $\begin{array}{l}\text { PASTE A: Silane-treated ceramic, TEGDMA, BisGMA, silane-treated silica, functionalized dimethacrylate } \\
\text { polymer, triphenylantimony }\end{array}$ & \multirow{2}{*}{1526500149} \\
\hline & $\begin{array}{c}\text { PASTE B: Silane-treated ceramic, TEGDMA, BisGMA, silane-treated silica, functionalized dimethacrylate } \\
\text { polymer, 2-benzotriazolyl-4-methylphenol, benzoyl peroxide }\end{array}$ & \\
\hline $\begin{array}{l}\text { Adper Single } \\
\text { Band } 2^{\mathrm{b}}\end{array}$ & $\begin{array}{c}\text { BisGMA, HEMA, UDMA, dimethacrylates, ethanol, water, camphorquinone, photoinitiators, polyalkenoic } \\
\text { acid copolymer, 5-nm silica particles }\end{array}$ & N688653 \\
\hline RelyX Unicem 2 ${ }^{\mathrm{b}}$ & $\begin{array}{l}\text { Base paste: silane-treated glass powder, 2-propenoic acid, 2-methyl-, reaction products with } \\
\text { 2-hydroxy-1,3-propanediyl dimethacrylate and phosphorus oxide, TEGDMA, silane-treated silica, sodium } \\
\text { persulfate, glass powder, tertbutyl peroxy-3,5,5-trimethylhexanoate, cooper acetate monohydrate } \\
\text { Catalyst paste: Silane-treated glass powder, substituted dimethacrylate, 1-benzyl-5-phenyl-barbic-acid, } \\
\text { calcium salt, silane--treated silica, sodium }\end{array}$ & 588286 \\
\hline \multirow[t]{2}{*}{ RelyX Ultimate ${ }^{b}$} & $\begin{array}{c}\text { Base paste: Silane--treated glass powder, 2-propenoic acid, 2-methyl-, reaction products with } \\
\text { 2-hydroxy-1,3-propanedyl dimethacrylate and phosphorus oxide, TEGDMA, silane--treated silica, oxide } \\
\text { glass chemicals, sodium persulfate, tertbutyl peroxy-3,5,5-trimethylhexanoate, } \\
\text { copper acetate monohydrate }\end{array}$ & \multirow{2}{*}{1516800384} \\
\hline & $\begin{array}{l}\text { Catalyst paste: Silane-treated glass powder, substituted dimethacrylate, 1,12-dodecane dimethacrylate, } \\
\text { silane--treated silica, 1-benzyl-5-phentyl-barbic-acid, calcium salt, sodium p-toluenesulfinate, 2-propenic } \\
\text { acid, 2-methyl-, di-2,1-ethanediyl ester, calcium hydroxide, titanium dioxide }\end{array}$ & \\
\hline $\begin{array}{l}\text { Scotchbond } \\
\text { Universal }^{b}\end{array}$ & $\begin{array}{l}\text { BisGMA, HEMA, decamethylene dimethacrylate, ethanol, water, silane-treated silica, 2-propenoic acid, } \\
\text { methacrylated phosphoric acid, copolymer of acrylic and itaconic acid, ethyl-4-dimethylaminobenzoat, } \\
\text { camphorquinone, (dimethylamino) ethyl methacrylate, methyl ethyl ketone }\end{array}$ & 1516800384 \\
\hline
\end{tabular}

\section{Dentin surface preparation}

All surfaces were cleaned with pumice/water at low speed.

RelyX ARC: etched with 37\% phosphoric acid (FGM, Joinville, Brazil) for $15 \mathrm{~s}$, washed and gently air-dried. Application of Adper Single Bond 2: agitated for $15 \mathrm{~s}$, gently air-dried for solvent evaporation and photopolymerized for $20 \mathrm{~s}$.

RelyX Unicem 2: no treatment.

RelyX Ultimate: application of Scotchbond Universal, agitated for $20 \mathrm{~s}$, and gently air-dried for $5 \mathrm{~s}$ to evaporate the solvent.

\section{Luting procedures}

The cement was hand-mixed and applied on the dentin surface. A standardized constant pressure of $2 \mathrm{~kg}$ was applied to the flat surface of the crowns using a customized metallic device. The specimens were light-cured with a high-intensity LED (3M ESPE, St. Paul, USA) unit calibrated at $1,900 \mathrm{~mW} / \mathrm{cm}^{2}$ for $40 \mathrm{~s}$ on each side for the RelyX ARC groups and for $20 \mathrm{~s}$ for the other luting cement groups. The bonded specimens were stored in distilled water for $24 \mathrm{~h}$ at $37^{\circ} \mathrm{C}$ to wait for the monomer conversion.

\section{Microtensile bond strength test}

After $24 \mathrm{~h}$, the specimens were vertically sectioned into serial slabs and further into sticks with a water-cooled diamond blade using a low-speed cutting saw. Sixteen samples $(n=16)$ with cross-sectional areas of approximately $0.90 \mathrm{~mm}^{2}$ were obtained for each group. The specimens were 
attached to the universal testing machine (DL-2000 EMIC, São José dos Pinhais, Brazil) device using cyanoacrylate (Super Bonder Gel, Loctite Brazil Ltda, São Paulo, Brazil) and stressed to failure with a low cell of $50 \mathrm{~N}$ and a crosshead speed of $0.5 \mathrm{~mm} / \mathrm{min}$. The failure load $(\mathrm{N})$ was recorded, and the surface area $\left(\mathrm{mm}^{2}\right)$ for each sample was used to calculate the $\mu$ TBS in MPa using Mtest software (T-Systems, São Paulo, Brazil).

Fractured specimens from each group $(n=16)$ were air-dried, mounted on metallic stubs, sputtered with a gold layer, and then examined under a scanning electron microscope (SEM) (Inspect F50, FEI, Marshall, USA) operated at $20 \mathrm{kV}$ with different magnifications (250x, 1000x and 2000x) to determine the failure modes. Failure modes were classified into five types: a) cohesive failure into resin cement, b) adhesive failure between dentin and adhesive or resin cement, c) adhesive failure between resin cement and restorative material, d) cohesive failure into restorative material, and e) mixed failure when more than one type occurred.

\section{Statistical analysis}

The $\mu$ TBS values were analyzed using a two-way ANOVA (restorative material $x$ adhesive strategy) and post hoc multiple comparisons using Tukey's test. $\mathrm{p} \leq 0.05$ was considered significant. The software used was SPSS v17 (SPSS Inc., Chicago, USA).

\section{Results}

The two-way ANOVA analysis revealed that the restorative material was significant $(p=0.001)$, the luting strategy was not significant ( $p=0.176)$, and the interaction effect was significant $(p=0.001)$ (Table 2).
Regardless of the luting strategy, a higher $\mu$ TBS was obtained with Lava Ultimate and Vita Enamic, which were significantly different from those of Vita Mark II and Vita Suprinity ( $p<0.05)$. For Vita Mark II, a higher $\mu$ TBS was obtained with RelyX Ultimate (15.38 MPa), which did not differ significantly from RelyX Unicem 2 (14.88 MPa). RelyX Unicem 2 did not differ significantly from RelyX ARC (12.17 MPa). For Vita Suprinity, there were no significant differences between the RelyX ARC (16.62 MPa), RelyX Unicem 2 (14.21 MPa) or RelyX Ultimate (12.81 MPa). For Vita Enamic, a higher $\mu$ TBS was obtained with RelyX Ultimate (32.88 MPa), which was not significantly different from that obtained with RelyX ARC (29.31 MPa). RelyX ARC was not significantly different from RelyX Unicem 2 (25.97 MPa). For Lava Ultimate, a higher $\mu$ TBS was obtained with RelyX Unicem 2 (32.93 MPa) and RelyX ARC (30.71 MPa), which were significantly different from that obtained with RelyX Ultimate (21.64 MPa).

The failure modes are represented in Figure 1. All groups obtained at least three types of failure. For Vita Mark II, the predominant failure mode was the cohesive failure into resin cement (Figure 2a). However, for Vita Suprinity, when RelyX ARC was used, the predominant failure mode was adhesive between dentin and Adper Single Bond 2 adhesive (Figure 2b), whereas for RelyX Unicem 2 and RelyX Ultimate, the failure mode was adhesive between resin cement and restorative material (Figure 2c). The main failure mode observed with Vita Enamic and RelyX Unicem 2 was adhesive between resin cement and restorative material, but for RelyX ARC and RelyX Ultimate, it was cohesive into resin cement. The predominant failure mode noted for Lava Ultimate with RelyX Unicem 2 and RelyX Ultimate was cohesive into resin cement. However, with RelyX ARC, it was the adhesive between dentin and the adhesive.

Table 2. The $\mu$ TBS mean (MPa) and standard deviations.

\begin{tabular}{lccc}
\hline Variable & RelyX ARC & RelyX Unicem 2 & RelyX Ultimate \\
\hline Vita Mark II & $12.17 \pm 2.08^{\mathrm{Bb}}$ & $14.88 \pm 3.61^{\mathrm{Cab}}$ & $15.38 \pm 4.05^{\mathrm{Ca}}$ \\
Vita Suprinity & $16.62 \pm 6.46^{\mathrm{Ba}}$ & $14.21 \pm 3.88^{\mathrm{Ca}}$ & $12.81 \pm 3.03^{\mathrm{Ca}}$ \\
Vita Enamic & $29.31 \pm 7.20^{\mathrm{Aab}}$ & $25.97 \pm 5.45^{\mathrm{Bb}}$ & $32.88 \pm 4.29^{\mathrm{Aa}}$ \\
Lava Ultimate & $30.71 \pm 4.42^{\mathrm{Aa}}$ & $32.93 \pm 6.63^{\mathrm{Aa}}$ & $21.64 \pm 6.02^{\mathrm{Bb}}$ \\
\hline
\end{tabular}

Mean values represented with the same superscript uppercase letters (column) and same lowercase letters (line) indicate no significant differences according to Tukey's test ( $p>0.05)$. 


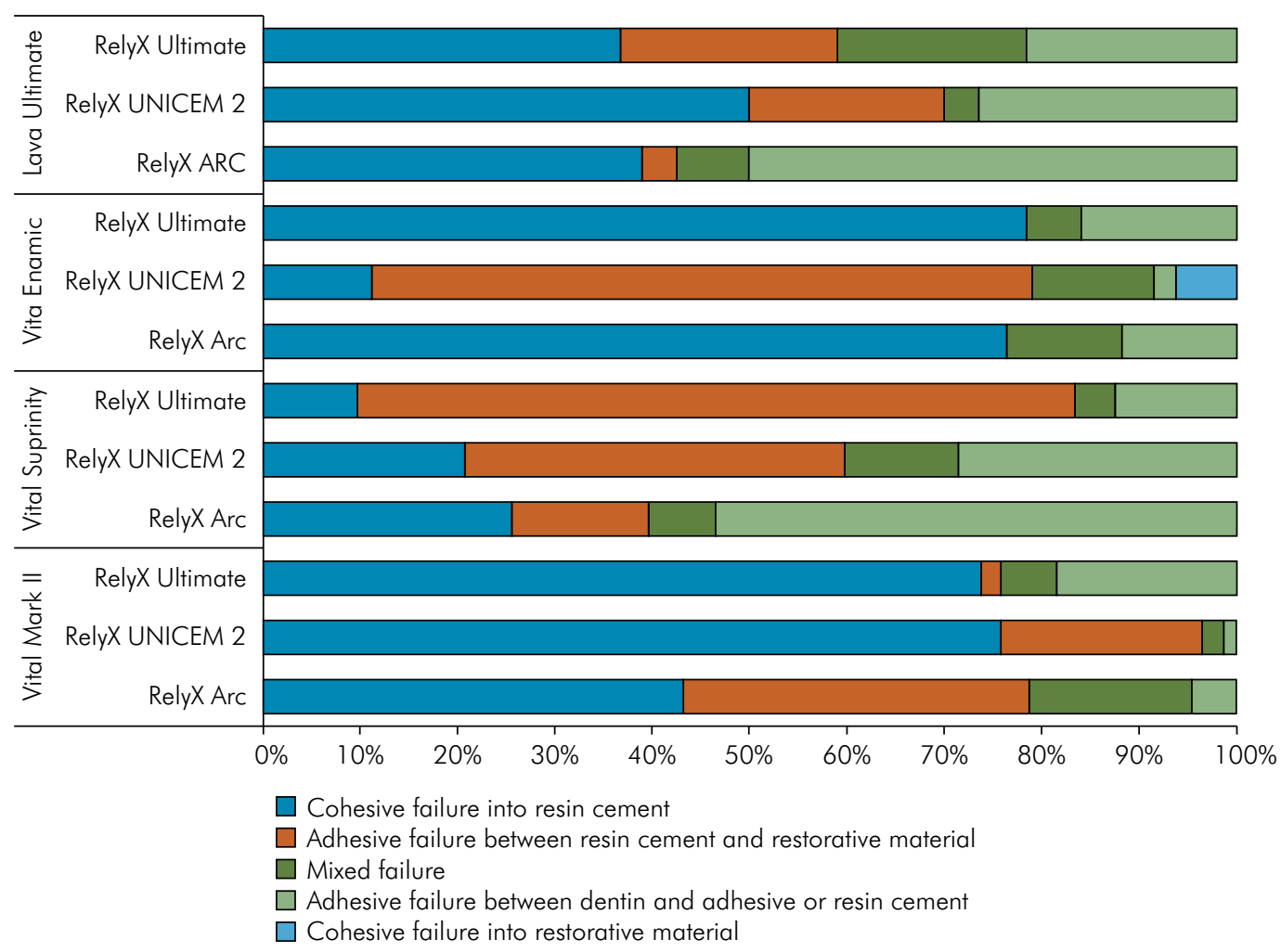

Figure 1. Failure mode analysis.

\section{Discussion}

The results showed that depending on the restorative material, the adhesive strategy influences the bond of CAD/CAM materials to dentin, rejecting the null hypothesis.

In the present study, two vitreous ceramics (Vita Mark II and Vita Suprinity), one polymer-infiltrated ceramic material (Vita Enamic), and a composite resin (Lava Ultimate) were evaluated. Vita Mark II, a feldspathic ceramic, was considered the control since this material has been on the market for several years. Additionally, the specimens submitted to $\mu$ TBS testing corresponded to a stick with a cross-sectional area of $\sim 0.90 \mathrm{~mm}^{2}$, and two main bond interfaces were formed at the time of luting the restorative materials on the dentin. The interfaces were a dentin-resinous agent interface and a resinous agent-restoration interface. Thus, the $\mu$ TBS values should be analyzed together with the types of failures that occurred in the different groups because the bond strength may be related to different interfaces.
Overall, Vita Enamic and Lava Ultimate obtained higher $\mu$ TBS values, which might be explained by the differences in the moduli of elasticity among the restorative materials. ${ }^{14}$ In previous studies, the moduli of elasticity of Vita Enamic and Lava Ultimate were $30.1 \mathrm{GPa}^{15}$ and $12.8 \mathrm{GPa},{ }^{4}$ respectively, which are similar to that of dentin $(16-20.3 \mathrm{GPa})^{6}$ and lower than those of Vita Mark II (57.2 GPa) ${ }^{16}$ and Vita Suprinity (70.44 GPa). ${ }^{9}$ Moreover, Vita Enamic and Lava Ultimate have similar moduli of elasticity to resin cements. ${ }^{17}$ The more brittle materials (Vita Mark II and Vita Suprinity) tend to start the fracture at the adhesive interface at lower values than the more resilient materials (Vita Enamic and Lava Ultimate) ${ }_{1}^{14}$ which could explain the lower $\mu$ TBS values of Vita Mark II and Vita Suprinity. Therefore, the differences in the moduli of elasticity among the materials could play a role in the bond strength results.

In an attempt to improve the bonding between resin cements and restorative materials, various surface treatments that facilitate chemical and micromechanical retention have been suggested. 

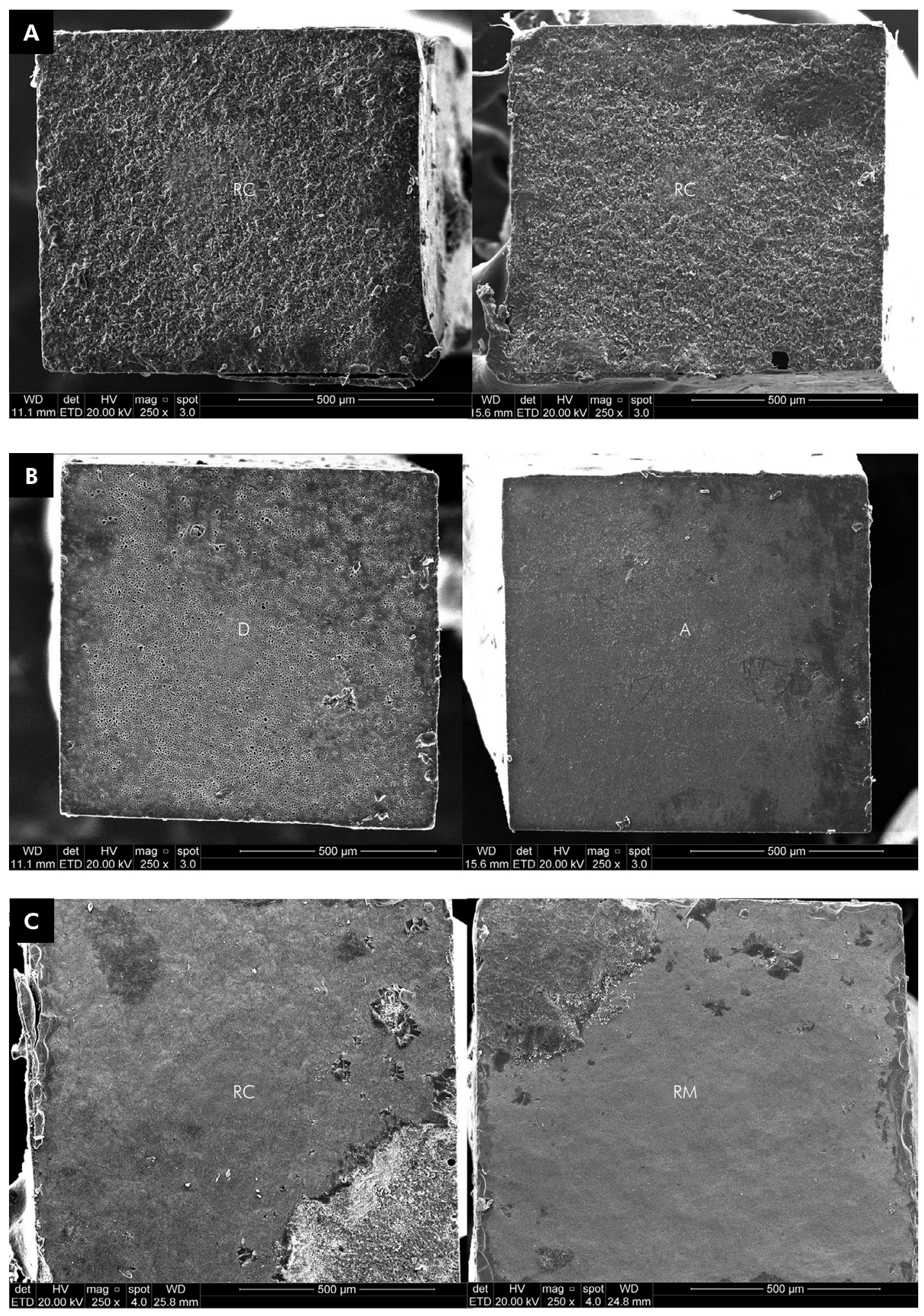

RC: resin cement; A: adhesive; D: dentin; RM :restorative material.

Figure 2. SEM images of the main failure modes obtained in the study. Left side: dentin side of the sample. Right side: restorative material side of the sample. a) cohesive failure into resin cement; b) adhesive failure between dentin and adhesive; c) adhesive failure between resin cement and restorative material. 
Increasing the surface energy of the composite by sandblasting, followed by silanization has been recommended as a predictable means to ensure retention between the resin cement and restorative composite. ${ }^{18,19,20}$ Furthermore, the silane coupling agent also contributes to $\mu \mathrm{TBS}$. It is an adhesion promoted between the organic (methacrylate monomers of the resin cement matrix) and inorganic (fillers of the indirect composite) surfaces and creates a chemical bond between the CAD/CAM resin blocks and resin cements. ${ }^{19,20,21,22}$ Due to these reasons, the Lava Ultimate samples were sandblasted and silanized in the present study. The failure mode for Lava Ultimate with RelyX Ultimate and RelyX Unicem 2 was predominantly cohesive into resin cement. Thus, the treatment of the dentin and of the restorative material was efficient, causing intrinsic rupture of the resin cement. However, cohesive failure into the resin cement did not necessarily provide higher bond strength values for the Lava Ultimate samples luted with RelyX Ultimate (21.64 MPa). When Lava Ultimate was luted with RelyX ARC, a high percentage of adhesive failure between the dentin and the Adper Single Bond 2 adhesive occurred, and the bond strength was higher (30.71 MPa) and did not differ significantly from that of RelyX Unicem 2 (32.93 MPa). For Lava Ultimate, the influence of micromechanical interlocking on the performance of the adhesive interface is particularly important since a high degree of conversion of polymers decreases the potential for chemical co-polymerization of any remaining free monomers with the monomers of the adhesive or resin cement. ${ }^{23}$

The adhesion of ceramic materials may be enhanced by increasing the surface energy, and it can improve the wettability of the adhesive or resin cement on the material surface. ${ }^{24}$ Thus, internal surface etching with hydrofluoric acid was performed according to the etching times recommended by the manufacturers of Vita Mark II, Vita Suprinity and Vita Enamic to create a reactive area that promotes resin infiltration. Vita Mark II and Vita Enamic were etched for $60 \mathrm{~s}$. In Vita Mark II, this surface treatment dissolved the glassy phase and promoted evident craters and pits, creating a honeycomb-like surface ideal for micromechanical retention. ${ }^{25}$ The chemical etching process can be explained by the preferential reaction of the hydrofluoric acid with the silica phase of the feldspathic ceramic (Vita Mark II) to form hexafluorosilicates. ${ }^{26}$ The topography of the etched surface of Vita Enamic was characterized by the persistent resin matrix, while the ceramic matrix was partially removed, and microporosities were formed on the surface. Consequently, the surface of the ceramic became rough and micromechanically retentive. ${ }^{23,27}$ Vita Suprinity was etched for $20 \mathrm{~s}$, and this surface treatment created a relatively smooth surface with small undercuts, which can be attributed to the specific microstructure with fine, densely packed crystals. ${ }^{27}$ Additionally, when the ceramic is etched, hydroxyl groups are exposed, and this group allows a chemical interaction with the silane coupling agent. ${ }^{28,29}$ Therefore, the samples were silanized in the present study.

When Vita Suprinity was luted with RelyX Unicem 2 or RelyX Ultimate, a high percentage of adhesive failure between the resin cement and restorative material was observed. Ramakrishnaiah et al. ${ }^{30}$ evaluated the extended etching time of Vita Mark II and Vita Suprinity and showed that the surface roughness and wettability of silica-based ceramics increased. Al-Thagafi et al. ${ }^{10}$ evaluated different etching protocols for Vita Suprinity and obtained higher values for the tribochemical silica coating with a silane coating. This finding indicates that the etching protocol for Vita Suprinity could be revised. Additionally, when Vita Suprinity was luted with RelyX ARC, the predominant failure mode was adhesive between the dentin and Adper Single Bond 2 adhesive. Thus, compared with Adper Single Bond 2, the bond strength of Scotchbond Universal with RelyX Ultimate and RelyX Unicem 2 self-adhesive resin cement performed better on the dentin. However, regardless of the failure modes obtained, the bond strength values did not differ significantly between the adhesive strategies for Vita Suprinity.

The failure mode was predominantly cohesive into resin cement for Vita Mark II luted with the three adhesive strategies and for Vita Enamic luted with RelyX ARC and RelyX Ultimate. Thus, the treatment to the dentin and to the restorative material was efficient, causing intrinsic rupture of 
the resin cement. Vita Enamic has a low percentage of organic matrix, which may contribute little to the chemical co-polymerization of free monomers with the monomers of the adhesive or resin cement. ${ }^{23}$ Therefore, it is assumed that micromechanical retention is important for the bond interface. However, when Vita Enamic was luted with RelyX Unicem 2, a high percentage of adhesive failure between the resin cement and restorative material occurred, having the lowest bond strength $(25.97 \mathrm{MPa})$ among the adhesive strategies. This result suggests the need to find alternative surface treatment protocols to stabilize this bond.

The present study showed similar $\mu$ TBS values between separate bottle of silane coupling agent and the universal adhesive, Scotchbond Universal, which contains in one solution silane, HEMA, MDP and Bis-GMA. ${ }^{31,32}$ However, previous studies reported that separate silane steps perform better than silane-containing universal adhesive, ${ }^{33}$ especially for long-term storage ${ }^{31}$ and thermocycling. ${ }^{32,34}$

The success of indirect restorations depends largely on the bonding system to ensure an effective and stable bond between the restorative material and dental substrates. ${ }^{35}$ Different bonding strategies were applied in the present study: total-etch adhesive, self-etch adhesive, and self-adhesive resin cement. This study demonstrated that the self-adhesive resin cement did not differ from those with adhesive application. This finding contributes to the desire for simplification of the luting procedure.

In the present study, the adhesive strategy effect was not significant. Suzuki et al. ${ }^{36}$ tested RelyX Unicem and RelyX ARC at $\mu$ TBS and recorded no significant differences. Hikita et al. ${ }^{37}$ evaluated the same strategy and concluded equal bond strength to dentin. In both studies, compared with the tested materials, the restorative material used was a direct resin composite with a lower modulus of elasticity.
Previous studies recorded different data from the present findings. ${ }^{19,38,39}$ The $\mu$ TBS values were higher for the total-etch than for the self-adhesive resin cement. These studies used a direct composite resin. A possible explanation for the observed difference between studies is the experimental design. While Frankenberger et al ${ }^{40}$ only evaluated the $\mu$ TBS between the luting cement and restorative material and did not mill the materials, in the present study, we evaluated the CAD/CAM material-luting cement-dentin system as well as the differences between etching protocols. Additionally, our samples were milled.

The present study showed that the use of Vita Enamic, Lava Ultimate, and Vita Suprinity is promising. Additionally, Vita Enamic and Lava Ultimate do not need crystallization in a dental furnace after milling, shortening the chair-side procedures. The $\mu$ TBS was evaluated $24 \mathrm{~h}$ after bonding. The main limitation of this study was not evaluating the effectiveness of $\mu$ TBS under long-term water storage and thermal-mechanical conditions. Therefore, further studies should be conducted to evaluate the mechanical and adhesive properties of these materials under long-term water storage and thermal aging conditions, even as clinical followups are recommended.

\section{Conclusion}

Within the limitations of this in vitro study, the following conclusions can be drawn:

a. The adhesive strategy with self-adhesive resin cement was comparable to that with conventional resin cement with total-etch or self-etch adhesive techniques in the bond of CAD/CAM materials to dentin,

b. Vita Enamic and Lava Ultimate showed higher immediate bond strength in comparison with Vita Mark II and Vita Suprinity.

\section{References}

\footnotetext{
1. Davidowitz G, Kotick PG. The use of CAD/CAM in dentistry. Dent Clin North Am. 2011;55(3):559-70. https://doi.org/10.1016/i.cden.2011.02.011
}

\footnotetext{
2. Li RW, Chow TW, Matinlinna JP. Ceramic dental biomaterials and CAD/CAM technology: state of the art. J Prosthodont Res. 2014;58(4):208-16. https://doi.org/10.1016/i.jpor.2014.07.003
} 
3. Duarte S Jr, Sartori N, Phark JH. Ceramic-reinforced polymers: CAD/CAM hybrid restorative materials. Curr Oral Health Rep. 2016;3(3):198-202. https://doi.org/10.1007/s40496-016-0102-2.

4. Chen C, Trindade FZ, de Jager N, Kleverlaan CJ, Feilzer AJ. The fracture resistance of a CAD/CAM Resin Nano Ceramic (RNC) and a CAD ceramic at different thicknesses. Dent Mater. 2014;30(9):954-62. https://doi.org/10.1016/j.dental.2014.05.018

5. Elsaka SE. Bond strength of novel CAD/CAM restorative materials to self-adhesive resin cement: the effect of surface treatments. J Adhes Dent. 2014;16(6):531-40.

6. Coldea A, Swain MV, Thiel N. Mechanical properties of polymer-infiltrated-ceramic-network materials. Dent Mater. 2013 Apr; 29(4):419:26. https://doi.org/10.1016/i.dental.2013.01.002.

7. Albero A, Pascual A, Camps I, Grau-Benitez M. Comparative characterization of a novel cad-cam polymer-infiltratedceramic-network. J Clin Exp Dent. 2015 Oct;7(4):e495-500. https://doi.org/10.4317/iced.52521

8. Della Bona A, Corazza PH, Zhang Y. Characterization of a polymer-infiltrated ceramic-network material. Dent Mater. 2014;30(5):564-9. https://doi.org/10.1016/j.dental.2014.02.019

9. Elsaka SE, Elnaghy AM. Mechanical properties of zirconia reinforced lithium silicate glass-ceramic. Dent Mater. 2016;32(7):908-14. https://doi.org/10.1016/i.dental.2016.03.013

10. Al-Thagafi R, Al-Zordk W, Saker S. Influence of surface conditioning protocols on reparability of CAD/CAM Zirconia-reinforced Lithium Silicate Ceramic. J Adhes Dent. 2016;18(2):135-41.

11. Hitz T, Stawarczyk B, Fischer J, Hämmerle CH, Sailer I. Are self-adhesive resin cements a valid alternative to conventional resin cements? A laboratory study of the long-term bond strength. Dent Mater. 2012;28(11):1183-90. https://doi.org/10.1016/i.dental.2012.09.006

12. Burgess JO, Ghuman T, Cakir D, Swift EJ Jr. Self-adhesive resin cements. J Esthet Restor Dent. 2010;22(6):412-9. https://doi.org/10.1111/j.1708-8240.2010.00378.x

13. Ferracane JL, Stansbury JW, Burke FJ. Self-adhesive resin cements - chemistry, properties and clinical considerations. J Oral Rehabil. 2011;38(4):295-314. https://doi.org/10.1111/j.1365-2842.2010.02148.x

14. El Zohairy AA, De Gee AJ, Mohsen MM, Feilzer AJ. Microtensile bond strength testing of luting cements to prefabricated CAD/CAM ceramic and composite blocks. Dent Mater. 2003;19(7):575-83. https://doi.org/10.1016/S0109-5641(02)00107-0

15. Homaei E, Farhangdoost K, Tsoi JK, Matinlinna JP, Pow EH. Static and fatigue mechanical behavior of three dental CAD/CAM ceramics. J Mech Behav Biomed Mater. 2016;59:304-13. https://doi.org/10.1016/i.jmbbm.2016.01.023 PMID:26896763
16. Swain MV, Coldea A, Bilkhair A, Guess PC. Interpenetrating network ceramic-resin composite dental restorative materials. Dent Mater. 2016;32(1):34-42. https://doi.org/10.1016/i.dental.2015.09.009 PMID:26454798

17. Ausiello P, Rengo S, Davidson CL, Watts DC. Stress distributions in adhesively cemented ceramic and resin-composite Class II inlay restorations: a 3D-FEA study. Dent Mater. 2004;20(9):862-72. https://doi.org/10.1016/i.dental.2004.05.001

18. Stawarczyk B, Basler T, Ender A, Roos M, Ozcan $M$, Hämmerle $C$. Effect of surface conditioning with airborne-particle abrasion on the tensile strength of polymeric CAD/CAM crowns luted with self-adhesive and conventional resin cements. J Prosthet Dent. 2012;107(2):94-101. https://doi.org/10.1016/S0022-3913(12)60031-6

19. Higashi M, Matsumoto M, Kawaguchi A, Miura J, Minamino T, Kabetani T et al. Bonding effectiveness of self-adhesive and conventional-type adhesive resin cements to CAD/CAM resin blocks. Part 1: effects of sandblasting and silanization. Dent Mater J. 2016;35(1):21-8. https://doi.org/10.4012/dmi.2015-234

20. Cura M, González-González I, Fuentes V, Ceballos L. Effect of surface treatment and aging on bond strength of composite resin onlays. J Prosthet Dent. 2016;116(3):389-96. https://doi.org/10.1016/i.prosdent.2016.02.016

21. Zaghloul H, Elkassas DW, Haridy MF. Effect of incorporation of silane in the bonding agent on the repair potential of machinable esthetic blocks. Eur J Dent. 2014;8(1):44-52. https://doi.org/10.4103/1305-7456.126240 PMID:24966745

22. D'Arcangelo C, Vanini L. Effect of three surface treatments on the adhesive properties of indirect composite restorations. J Adhes Dent. 2007;9(3):319-26.

23. Eldafrawy M, Ebroin MG, Gailly PA, Nguyen JF, Sadoun MJ, Mainjot AK. Bonding to CAD-CAM composites: an interfacial fracture toughness approach. J Dent Res. 2017::22034517728714. https://doi.org/10.1177/0022034517728714

24. Jardel V, Degrange M, Picard B, Derrien G. Surface energy of etched ceramic. Int J Prosthodont. 1999;12(5):415-8.

25. Venturini AB, Prochnow C, Rambo D, Gundel A, Valandro LF. Effect of hydrofluoric acid concentration on resin adhesion to a feldspathic ceramic. J Adhes Dent. 2015;17(4):313-20. https://doi.org/10.3290/i.jad.a34592

26. Chen JH, Matsumura $H$, Atsuta M. Effect of different etching periods on the bond strength of a composite resin to a machinable porcelain. J Dent. 1998;26(1):53-8. https://doi.org/10.1016/S0300-5712(96)00078-4 PMID:9479926

27. Hu M, Weiger R, Fischer J. Comparison of two test designs for evaluating the shear bond strength of resin composite cements. Dent Mater. 2016;32(2):223-32. https://doi.org/10.1016/i.dental.2015.11.023 PMID:26723840

28. Lung CY, Matinlinna JP. Aspects of silane coupling agents and surface conditioning in dentistry: an overview. Dent Mater. 2012;28(5):467-77. https://doi.org/10.1016/j.dental.2012.02.009 
29. Özcan M, Volpato CA. Surface conditioning protocol for the adhesion of resin-based materials to glassy matrix ceramics: how to condition and why? J Adhes Dent. 2015;17(3):292-3.

30. Ramakrishnaiah R, Alkheraif AA, Divakar DD, Matinlinna JP, Vallittu PK. The effect of hydrofluoric acid etching duration on the surface micromorphology, roughness, and wettability of dental ceramics. Int J Mol Sci. 2016;17(6):E822. https://doi.org/10.3390/ijms17060822

31. Makishi P, André CB, Silva JL, Bacelar-Sá R, Correr-Sobrinho $L$, Giannini M. Effect of storage time on bond strength performance of multimode adhesives to indirect resin composite and lithium disilicate glass ceramic. Oper Dent. 2016;41(5):541-51. https://doi.org/10.2341/15-187-L

32. Lee Y, Kim JH, Woo JS, Yi YA, Hwang JY, Seo DG. Analysis of self-adhesive resin cement microshear bond strength on leucite-reinforced glass-ceramic with/without pure silane primer or universal adhesive surface treatment. BioMed Res Int. 2015;2015:361893. https://doi.org/10.1155/2015/361893

33. Gómez FM, De Góez MF. Effect of different silane-containing solutions on glass-ceramic/cement bonding interacting with dual-cure resin cements. ODOVTOS Int J Dental. 2014;16:87-105.

34. Kim RJY, Woo JS, Lee IB, Yi YA, Hwang JY, Seo DG. Performance of universal adhesive on bonding to leucite-reinforced ceramic. Biomater Res. 2015; 22:19:11. https://doi.org/10.1186/s40824-015-0035-1.

35. Manso AP, Silva NR, Bonfante EA, Pegoraro TA, Dias RA, Carvalho RM. Cements and adhesives for all-ceramic restorations. Dent Clin North Am. 2011;55(2):311-32. https://doi.org/10.1016/i.cden.2011.01.011

36. Suzuki TY, Godas AG, Guedes AP, Catelan A, Pavan S, Briso $A L$ et al. Microtensile bond strength of resin cements to caries-affected dentin. J Prosthet Dent. 2013;110(1):47-55. https://doi.org/10.1016/S0022-3913(13)60339-X

37. Hikita K, Van Meerbeek B, De Munck J, Ikeda T, Van Landuyt $K$, Maida $T$ et al. Bonding effectiveness of adhesive luting agents to enamel and dentin. Dent Mater. 2007;23(1):71-80. https://doi.org/10.1016/i.dental.2005.12.002

38. Fuentes MV, Escribano N, Baracco B, Romero M, Ceballos L. Effect of indirect composite treatment microtensile bond strength of self-adhesive resin cements. J Clin Exp Dent. 2016;8(1):e14-21.

39. Viotti RG, Kasaz A, Pena CE, Alexandre RS, Arrais $C A$, Reis AF. Microtensile bond strength of new selfadhesive luting agents and conventional multistep systems. J Prosthet Dent. 2009;102(5):306-12. https://doi.org/10.1016/S0022-3913(09)60180-3

40. Frankenberger R, Hartmann VE, Krech M, Krämer N, Reich S, Braun $A$ et al. Adhesive luting of new CAD/CAM materials. Int J Comput Dent. 2015;18(1):9-20. 


\section{Microtensile bond strength of CAD/CAM materials to dentin under different adhesive strategies. Braz Oral Res. 2017;31:e109.}

Where is read:

Julieta Gomes TAVARES(c)

Ana Maria SPOHR ${ }^{(c)}$

(a) Pontifícia Universidade Católica da Universidade Federal do Rio Grande do Sul - PUC-RS, School of Dentistry, Graduate Program, Porto Alegre, RS, Brazil.

It should read:

Julieta Gomes TAVARES(a)

Ana Maria SPOHR ${ }^{(a)}$

(a)Pontifícia Universidade Católica do Rio Grande do Sul - PUC-RS, School of Dentistry, Porto Alegre, RS, Brazil.

Disconsider:

(c) Pontifícia Universidade Católica da Universidade Federal do Rio Grande do Sul - PUC-RS, School of Dentistry, Clinical Department, Porto Alegre, RS, Brazil. 